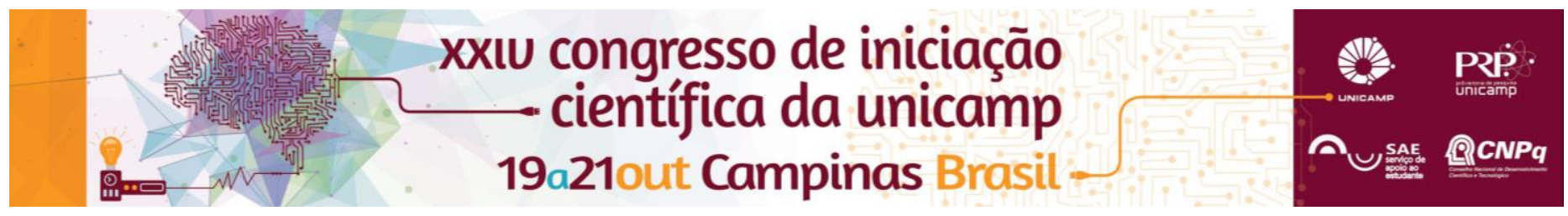

\title{
Estudo Retrospectivo do Valor Prognóstico da Ressonância Cardíaca em Pacientes com Cardiopatias
}

\author{
Nathalia C. Valiero, Pedro Jallad, Juliana Novaes, Amanda Fortunato, Otavio R. Coelho-Filho.
}

\section{Resumo}

A quantificação do espaço extracelular (ECV), um novo marcador de fibrose intersticial no miocárdio, através de medidas de T1 pela ressonância cardíaca magnética, identificou pacientes com miocardiopatia não isquêmica com elevado risco de eventos cardiovasculares, sendo um robusto fator nessa população.

Palavras-chave: Cardiopatias, Ressonância Magnética Cardíaca, Fibrose.

\section{Introdução}

A insuficiência cardíaca (IC) é uma condição altamente prevalente e que mantém elevada morbidade e mortalidade a despeito de recentes e grandes avanços diagnósticos e terapêuticos[1]. A ressonância magnética cardíaca (RMC) emerge como um método com elevada acurácia para avaliação da morfologia e função cardíacas, além de fornecer dados a respeito da presença de cicatriz/infarto focais através da técnica do realce tardio(RT)[2]. Apesar do RT ser uma técnica validada para avaliar fibrose miocárdica, essa técnica não consegue detectar a fibrose intersticial difusa. Nosso grupo descreveu uma técnica para avaliação do espaço extracelular (ECV), um marcador de fibrose intersticial no miocárdio, através de medidas de T1 antes e depois da administração de contraste, mesmo na ausência de RT[35], que apresenta grande potencial de aplicação nas miocardiopatias com implicações não apenas diagnósticas, mas possivelmente prognósticas.

Objetivo: Quantificar o impacto prognóstico das variáveis oriundas da RMC em pacientes com miocardiopatia nãoisquêmica com FEVE<35\%. Avaliar se o ECV apresenta fator prognóstico complementar a outros fatores clínicos e da RMC.

\section{Resultados e Discussão}

Foram identificados 127 pacientes (idade-média: $52 \pm 12$ anos, $43 \%$ do sexo feminino, IMC $26 \pm 6 \mathrm{~kg} / \mathrm{m}^{2}$ ) com miocardiopatia não-isquêmica que realizaram exame de RMC (3-Tesla Achieva, Philips Medical Systems, Best, The Netherlands) com obtenção da morfologia e função cardíaca além do RT após administração de gadolínio $(0,2 \mathrm{mmol} / \mathrm{kg})$. Foram também obtidos dados para quantificação ECV, um marcador da fibrose intersticial miocárdio. A avaliação da morfologia e função mostrou que coorte era constituída por pacientes com FEVE acentuadamente reduzida $(27 \pm 9 \%), \quad$ FEVD moderadamente reduzida $(41 \pm 17 \%)$ com aumento do átrio esquerdo $(49 \pm 8 \mathrm{~mm})$, aumento da massa do VE $(159 \pm 55 \mathrm{~g})$ e com ECV elevado $(0,43 \pm 0,09)$. 78 pacientes $(61 \%)$ apresentaram RT com padrão não isquêmico. Após um seguimento mediano de 375 dias, 32 eventos ocorreram, incluindo 18 admissões por IC, 10 transplantes de coração e 4 mortes cardíacas. Utilizando o método de regressão tipo cox, verificamos que as únicas vaiáveis que apresentaram associação com os desfechos foram: classe da NYHA (HR: 2,14; IC 95\%: 1,2-3,7; p=0,007), ECV (HR: 2062; IC 95\%: 8,7-488862,8; $p=0,006$ ), FC em repouso (HR: 1,05; IC 95\%: 1,05-1,08; p=0,003). A associação do ECV com os desfechos manteve significância estatística mesmo após o ajuste para idade, sexo, FEVE e FEVD. A avaliação da probabilidade de sobrevida sem desfechos pelo método de Kaplan Meier (figura 1), estratificada por tercis do ECV (tercil-inferior: 0,12-0,39, tercil-médio:0,390,47 , tercil-superior $>0,47$ ) revelou que quanto maior o tercil menor foi probabilidade de seguimento livre de eventos (Log-Rank<0.001, para todas as comparações).

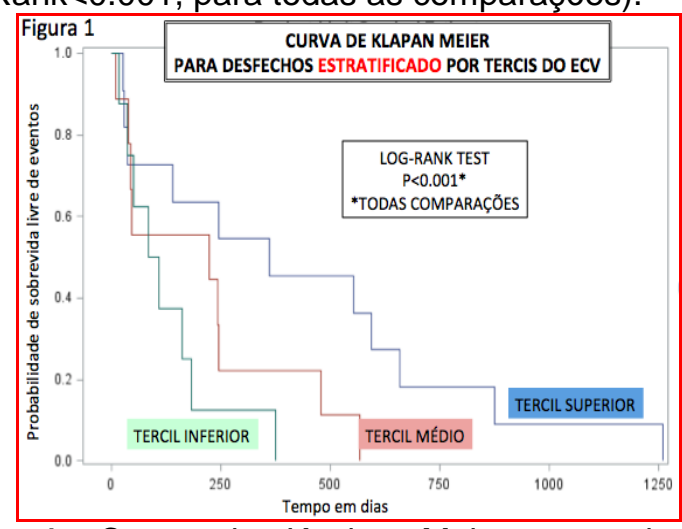

Figura 1. Curva de Kaplan Meier para desfechos estratificado por tercis do ECV.

\section{Conclusões}

O presente estudo mostrou que em pacientes com IC com FEVE reduzida não isquêmica a FC de repouso, a classe do NYHA e o ECV determinado pela RMC foram fatores significativamente associados com o desfecho combinado de morte, admissão por IC e transplante cardíaco. Dentre esses fatores o ECV foi o que mais fortemente se associou com os eventos, mantendo essa associação mesmo quando ajustado para fatores clássicos com idade, sexo, FEVE e FEVD. Em pacientes com IC com FEVE reduzida o ECV pode ser muito útil na melhora da estratificação de risco, identificando os pacientes com IC que podem mais se beneficiar de medidas terapêuticas mais invasivas e custosas como implante de cardio-desfibrilador ou monitor de pressão da artéria pulmonar.

\section{Referências}

1. Bocchi, E.A., et al., [III Brazilian Guidelines on Chronic Heart Failure]. Arq Bras Cardiol, 2009. 93(1 Suppl 1): p. 3-70.

2. Sara, L., et al., [II Guidelines on Cardiovascular Magnetic Resonance and Computed Tomography of the Brazilian Society of Cardiology and the Brazilian College of Radiology]. Arq Bras Cardiol, 2014. 103(6 Suppl 3): p. 1-86.

3. Coelho-Filho, O.R., et al., Role of transcytolemmal water-exchange in magnetic resonance measurements of diffuse myocardial fibrosis in hypertensive heart disease. Circ Cardiovasc Imaging, 2013. 6(1): p. 134-41.

4. Coelho-Filho, O.R., et al., Quantification of cardiomyocyte hypertrophy by cardiac magnetic resonance: implications for early cardiac remodeling. Circulation, 2013. 128(11): p. 1225-33.

5. Neilan, T.G., et al., Myocardial extracellular volume fraction from T1 measurements in healthy volunteers and mice: relationship to aging and cardiac dimensions. JACC Cardiovasc Imaging, 2013. 6(6): p. 672-83. 